\title{
The time has come for next-generation sequencing in routine diagnostic workup in hematology
}

\author{
Torsten Haferlach \\ MLL Munich Leukemia Laboratory, Munich, Germany \\ E-mail: TORSTEN HAFERLACH - torsten.haferlach@mll.com
}

doi:10.3324/haematol.2020.270504

$I^{n}$ $\mathrm{n}$ this issue of Haematologica, Vantyghem et al. ${ }^{1}$ demonstrate, in a real-life setting, how the application of a customized next-generation sequencing (NGS) panel can support routine diagnostics, deliver prognostic information, and may even directly influence the choice of therapy towards a more personalized treatment.

The diagnostic workup for patients with unexplained cytopenia or proliferative blood counts currently follows specific clinical procedures: After a deep dive into the patient's history and an examination of his or her complete blood count, a peripheral blood smear is evaluated microscopically. At the same time, several medical imaging techniques, such as ultrasound or computed tomography scans, are used to determine the plausible cause of the observed signs and symptoms. If a malignant hematologic disorder is suspected, a bone marrow aspirate and biopsy are often the next steps. The material obtained is then analyzed morphologically, cytogenetically, and histologically. ${ }^{2}$ However, even combining the results of the various investigations (cytomorphology, cytochemistry, cytogenetics, fluorescence in situ hybridization, histology, immunohistology, and multiparameter flow cytometry) does not guarantee a precise diagnosis in all cases, e.g., a malignant hematologic disorder or reactive/normal/benign conditions. For patients without a firm diagnosis, the treatment options dwindle down to 'watch-and-wait' often accompanied by the application of more diagnostic techniques (e.g., magnetic resonance imaging, positron emission tomography), and "reevaluation in 3 months if blood counts remain abnormal".

However, with the increased importance and acceptance of molecular genetics in the last decade, substantial progress has been achieved. The work of various research groups has made it possible to define the landscape of molecular findings for the diagnosis of myelodysplastic syndrome, myeloproliferative neoplasm, myelodysplastic syndrome/myeloproliferative neoplasm and severe aplastic anemia. ${ }^{3.7}$ This not only fosters diagnostic clarity but also has an increasing impact on prognosis and therapeutic options, including targeted treatment.

Vantyghem et al. divided their cohort of 177 patients with suspected chronic myeloid malignancies into two overlapping groups to exemplify the clinical impact of targeted sequencing to confirm or discard a suspected diagnosis (group A) and to assess the therapeutic consequences of somatic mutations (group B). A panel of 34 genes was used to search for clonal hematopoiesis in group A patients for whom the gold standard routine workup had not yielded a conclusive diagnosis. Cytogenetic chromosomal banding analysis, carried out in $86 \%$ of the cases of group A, revealed a normal karyotype in $72 \%$ of the tested patients. Only in $8 \%$ of the

cases was a cytogenetic aberration found that might have been useful for diagnosis and prognosis according to international risk scoring systems. However, the identification of clonal hematopoiesis in $33 \%$ of the patients confirmed the diagnosis in 31 patients, whereas the absence of clonal hematopoiesis ruled out a chronic myeloid malignancy in 47 patients. Moreover, in group B prognostic mutations were identified in $33 \%$ of the patients, with this prognostic information affecting treatment choices in 18 cases.

The results clearly demonstrate the advantages of molecular approaches for, but not limited to, patients without a firm diagnosis from classical investigations. The authors concluded that for those patients cell pellets or DNA should be stored at first investigation to provide the possibility of any kind of molecular investigations at a later time point.

The approach described also has a socio-economic impact: any repeated testing procedures submit patients, who are already stressed by the uncertainty of their disease state, to additional discomfort and put a strain on the healthcare system by causing extra costs and consuming valuable resources, such as the time of doctors and laboratories.

On the other hand, any kind of targeted treatment, guided by NGS results, will not only serve patient's needs best but will also avoid treatment costs for suboptimal outcomes. Depending on molecular genetic findings, treatment modalities might be initiated, altered, postponed or in some cases even stopped. The limit of the theranostic impact of NGS is far from being reached. With the establishment of multicenter, large-scale sequencing projects, more information on frequency and diversity of germline and especially somatic variants across different disease entities will be gained, improving variant interpretation and increasing the number of potentially actionable targets. ${ }^{8}$ In the present study, Vantyghem et al. identified multiple variants in genes associated with myeloid malignancies but due to the limited amount of information for these variants, they had to be classified as variants of unknown significance, excluding them (so far) from the therapeutic decision-making process.

The increase in knowledge will also lead to optimized bioinformatics workflows to improve the sensitivity and specificity of variant detection and to decrease the influence of technical noise, which results in technical artifacts that currently have to be removed through painstaking manual efforts. Various collaborations are underway to create consensus somatic pathogenicity datasets for standardized variant interpretation. ${ }^{9}$ For high-throughput laboratories, parallelization of analysis pipelines will help to 


\section{Cohort}

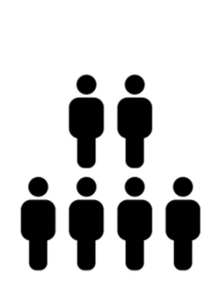

$\mathrm{n}=177$

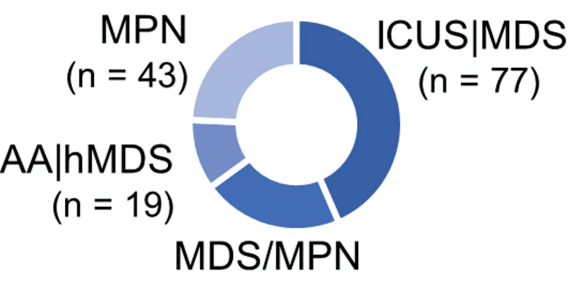

$(\mathrm{n}=38)$
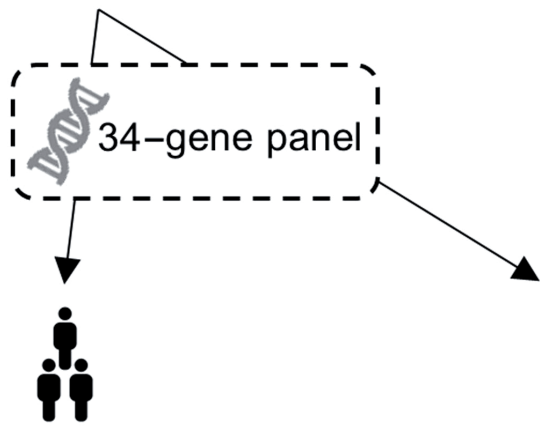

Group A

$(n=94)$

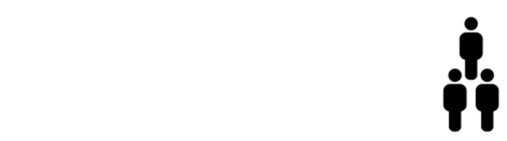

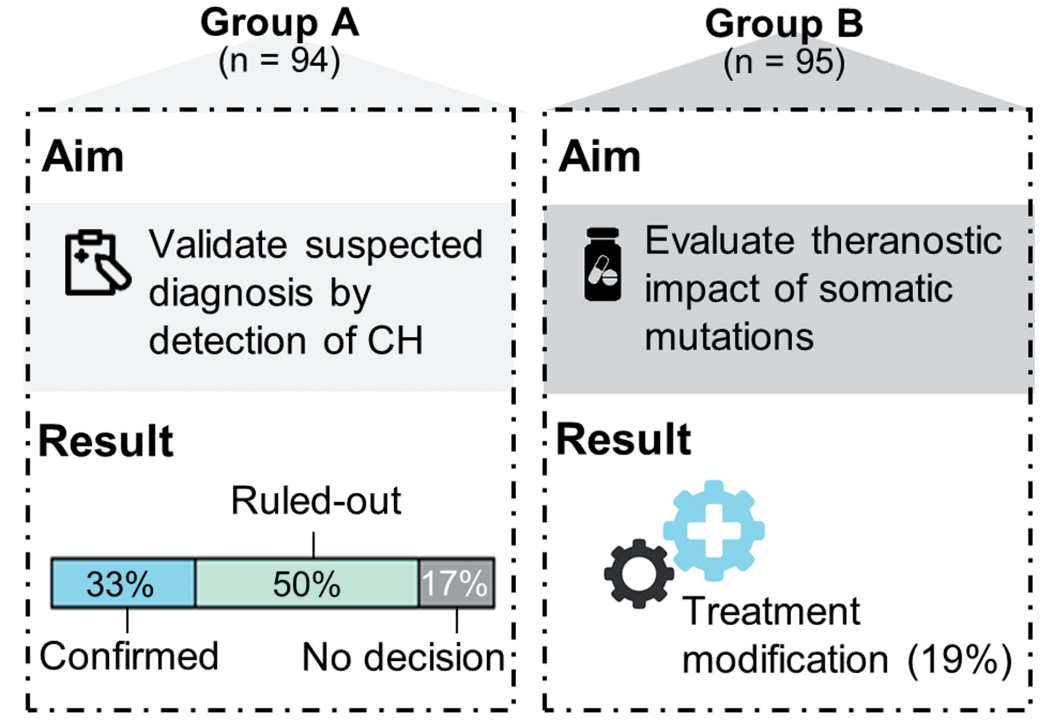

keep turn-around times within a reasonable time frame, allowing a personalized molecular analysis for every patient before treatment starts. Turn-around times might be further reduced by using machine-learning techniques for speedy and accurate evaluation of test results. ${ }^{10}$

We must serve our patients to the best of our capacity by applying state-of-the-art methodologies to extend and supplement standard diagnostic criteria. Thus, any new data, any new scientific finding and any new assay needs to be evaluated, tested, and validated based on the best of the current available guidelines. ${ }^{11,12}$ In addition, analysis pipelines must be benchmarked to ensure flawless behavior with acceptable error margins. ${ }^{13}$ Following rigorous testing and scientific validation, we have the mandate to integrate new techniques with diagnostic, prognostic or therapeutic benefit into clinical routine practice as early as possible. Vantyghem et al. showed that in $83 \%$ of patients, a mutational profile was useful for making an integrated final diagnosis. In 19\% the additional information gained by NGS data had prognostic impact and led to treatment modifications.
If we want to reduce the level of uncertainty in the diagnosis of hematologic malignancies, NGS might provide us with the necessary additional information already today. ${ }^{14,15}$ Even if NGS is still comparably expensive, the confidence gained regarding a correct, final diagnosis, which reduces a patient's fear and prevents wrong treatment, justifies its application already now.

\section{Disclosures}

TH is part owner of MLL Munich Leukemia Laboratory

\section{References}

1. Vantyghem S, Peterlin P, Thépot $S$, et al. Diagnosis and prognosis are supported by integrated assessment of next-generation sequencing in chronic myeloid malignancies. A real-life study. Haematologica. 2021;106(3):701-707.

2. Swerdlow SH, Campo E, Harris NL, et al. WHO Classification of Tumours of Haematopoietic and Lymphoid Tissues. Revised fourth edition. Lyon: IARC; 2017.

3. Haferlach T, Nagata Y, Grossmann V, et al. Landscape of genetic lesions in 944 patients with myelodysplastic syndromes. Leukemia. 
2014·28(2):241-247

4. Yoshizato T, Dumitriu B, Hosokawa K, et al. Somatic mutations and clonal hematopoiesis in aplastic anemia. N Engl J Med. 2015;373(1):35-47

5. Palomo L, Meggendorfer M, Hutter S, et al. Molecular landscape and clonal architecture of adult myelodysplastic/myeloproliferative neoplasms. Blood. 2020;136(16):1851-1862.

6. Schischlik F, Kralovics R. Mutations in myeloproliferative neoplasms their significance and clinical use. Expert Rev Hematol. 2017;10(11):961-973

7. Valent P, Kern W, Hoermann G, et al. Clonal hematopoiesis with oncogenic potential (CHOP): separation from CHIP and roads to AML. Int J Mol Sci. 2019;20(3):789.

8. Meggendorfer M, Walter W, Haferlach T. WGS and WTS in leukemia: a tool for diagnostics? Best Pract Res Clin Haematol. 2020;33(3):101190.

9. Nadarajah N, Wagner A, Bejar R, et al. Creating a variant database for the American Society of Hematalogy by consensus variant clas- sification of common genes associated with hematologic malignancies. Blood. 2020;136(Suppl 1):4-5.

10. Radakovich N, Nagy M, Nazha A. Machine learning in haematological malignancies. Lancet Haematol. 2020;7(7):e541-e550

11. Matthijs G, Souche E, Alders M, et al. Guidelines for diagnostic nextgeneration sequencing. Eur J Hum Genet. 2016;24(1):2-5.

12. Jennings LJ, Arcila ME, Corless C, et al. Guidelines for validation of next-generation sequencing-based oncology panels: a joint consensus recommendation of the Association for Molecular Pathology and College of American Pathologists. J Mol Diagn. 2017;19(3):341-365.

13. Mangul S, Martin LS, Hill BL. Systematic benchmarking of omics computational tools. Nat Commun. 2019;10(1):1-11

14. Malcovati L, Galfi A, Travaglino E, et al. Clinical significance of somatic mutation in unexplained blood cytopenia. Blood. 2017;129(25):3371-3378.

15. Baer C, Pohlkamp C, Haferlach C, et al. Molecular patterns in cytopenia patients with or without evidence of myeloid neoplasm-a comparison of 756 cases. Leukemia. 2018; 32(10):2295-2298.

\title{
Recognition of thrombotic risk of thrombocytosis in iron deficiency
}

\author{
Hanny Al-Samkari, ${ }^{1}$ Craig M. Kessler ${ }^{2}$ and Michael Auerbach ${ }^{2}$ \\ ${ }^{1}$ Division of Hematology, Massachusetts General Hospital, Harvard Medical School, Boston, MA and 'Department of Medicine, \\ Georgetown University School of Medicine, Washington, DC, USA \\ E-mail: MICHAEL AUERBACH - mauerbachmd@abhemonc.com
}

doi:10.3324/haematol.2020.270496

I n 1999, Loo and Beguin examined the effect of recombinant human erythropoietin on megakaryopoiesis, noting that iron-replete and iron-overloaded rats treated with recombinant human erythropoietin demonstrated a one-third decreased duration of thrombocytosis compared with normal rats. ${ }^{1}$ The putative explanation given for the increased duration in the normal rats was erythropoietin-induced, iron-deficient erythropoiesis. This provocative hypothesis was subsequently supported by the findings of a post-hoc analysis ${ }^{2}$ of a large prospective study of oral versus intravenous or no iron as adjunctive therapy for erythropoiesis-stimulating agent-treated patients with cancer and chemotherapy-induced anemia. ${ }^{3}$ This study demonstrated that intravenous, but not oral, iron repletion resulted in marked improvements in hemoglobin and hematopoietic responses and also re-demonstrated the known associations of increased thromboses and thrombocytosis among patients with cancer receiving erythropoiesis-stimulating agents. Of 187 patients treated with an erythropoiesis-stimulating agent, 19 developed 29 venous thromboembolic events and those with platelet counts greater than 350,000 per microliter were three times more likely to develop venous thromboembolism. Notably, there was a reduced incidence of thrombocytosis and thrombotic complications in those treated with intravenous iron compared with those who were not.

Further corroborating evidence pointing to iron-restricted erythropoiesis resulting in reactive thrombocytosis and thrombosis was derived from a recently published study describing the rate, predictors, and thrombotic complications of thrombocytosis in patients with irondeficiency anemia. ${ }^{4}$ Utilizing queries of an institutional database containing clinical information on more than six million patients over a 40-year time period (1979-2019), supported by extensive manual chart reviews, thrombocytosis was observed in $32.6 \%$ of patients with iron-deficiency anemia, in whom $15.8 \%$ developed thrombotic complications. In contrast, thromboses occurred in $7.8 \%$ of those with iron deficiency but without thrombocytosis (Figure 1).

The etiological explanation for why iron-restricted erythropoiesis increases hypercoagulability has remained somewhat obscure. In this issue of Haematologica, Jimenez and colleagues describe an animal model of iron deficiency, using Sprague-Dawley rats, which could offer unique opportunities to explore multiple pathophysiological mechanisms for thrombocytosis and venous thrombogenesis. ${ }^{5}$ Furthermore, using sensitive histological and sophisticated high-frequency ultrasound techniques, the investigators were able to track the development and progression of thrombus formation. They were then able to correlate those changes with the generation of ex vivo biomarkers of platelet activation and indicators of global hemostasis. The potential use of this rat model to detect important targets that could be exploited therapeutically to mitigate the association of iron deficiency with thrombocytosis and thromboembolism is promising and encouraging.

In their elegant description of iron deficiency-induced augmentation of coagulability on thromboelastography and hemostasis following rat tail resection, prominent correlates of increased platelet adhesion and aggregation were clearly described. Using in vitro assays of platelet aggregation, the authors were able to postulate that not only is platelet activation an ongoing process in inflammatory conditions but in iron deficiency as well. Importantly, given the frequent comorbidity of iron defi- 